\title{
Protection of Passive Optical Networks by Using Ring Topology and Tunable Splitters
}

\author{
Pavel Lafata
}

\begin{abstract}
This article proposes an innovative method for protecting of passive optical networks (PONs), especially the central optical unit - optical line termination (OLT). PON networks are typically used in modern high-speed access networks, but there are also several specific applications, such as in business, army or science sector, which require a complex protection and backup system against failures and malfunctions. A standard tree or star topologies, which are usually used for PON networks, are significantly vulnerable mainly against the malfunctions and failures of OLT unit or feeder optical cable. The method proposed in this paper is focused on forming PON network with ring topology using passive optical splitters. The main idea is based on the possibility of placing both OLT units (primary and secondary) on the opposite sides of the ring, which can potentially increase the resistance of network. This method is described in the article and scenarios and calculations using symmetric or tunable asymmetric passive optical splitters are included as well.
\end{abstract}

Keywords-Passive Optical Networks, Passive Optical Splitters, Protection, Ring Topology, Tunable Splitters.

\section{INTRODUCTION}

The passive optical networks (PONs) are usually used mostly as modern high-speed last-mile access networks. The present generation of PONs, such as XG-PON according to ITU-T G.987 [1] or 10GEPON in IEEE 802.3av [2] recommendations, offers typical shared transmission capacity up to $10 \mathrm{Gbps}$ for up to 128 connected users for a distances such as 20 or $40 \mathrm{~km}$ [3]. Other special applications of PONs can include local backbone data networks with optimized topology and optical distribution network with optimized bus topology [4]. However, PONs can be also used for several specific applications in industry, business, office or army sectors, which usually require higher level of protection and availability using protection and backup mechanisms. These applications typically require high network availability together with the guarantee of maximum functionality of the whole network infrastructure. That is

Manuscript received October 26, 2012. This work was supported in part by the Grant no. VG20102015053 - The modern structure of photonic sensors and new innovative principles for intrusion detection systems, integrity and protection of critical infrastructure - GUARDSENSE and also by the grant no. SGS10/275/OHK3/3T/13 - Collaborative Research in the Field of Optical Components, Networks and Digital Signal Processing for Telecommunications.

Ing. Pavel Lafata, Ph.D. is an assistant professor at the Department of Telecommunication Engineering, Faculty of Electrical Engineering, Czech Technical University in Prague, Czech Republic. (phone: +42022435 4088; e-mail: lafatpav@ffel.cvut.cz). why it is necessary to develop simple and efficient methods of protecting the critical optical units in PONs as well as of the whole optical distribution network.

One of these problems consists of protecting the optical line termination (OLT), which is a central optical unit of the whole PON. This unit provides mainly communication controlling, management and servicing functions of the whole network and it also connects the PON network into the backbone telecommunication infrastructure [3]. It is obvious that its potential failure or malfunction would surely result into a collapse of the whole PON.

A typical optical distribution network usually has a star topology with a single branching point, or a tree topology with several branching points [3], which makes the methods for OLT backup difficult. While in case of a star or a tree topology all optical fibers are concentrated into one single central point, backup (secondary) OLT can be placed only into the same place as the primary one, as illustrated in following Fig. 1. Such backup cannot be very reliable and the whole infrastructure is still vulnerable to many situations, e.g. global power failure, floods, terrorist action, breaking of feeder optical cable etc.

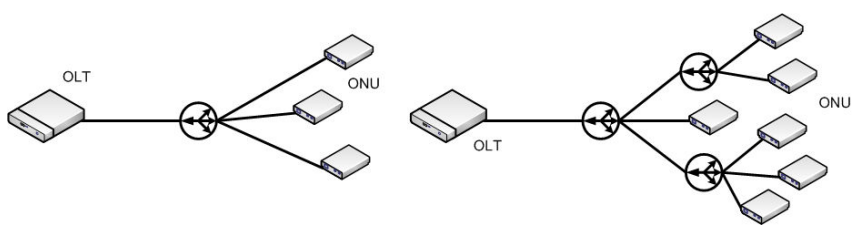

Fig. 1. Typical star and tree topology in case of PONs.

That is why the optimal topology for the critical application of PON is a ring topology [5], but presented applications require special optical network units (ONU) with optical switches and others nonstandard enhancements. However, ring topology could be also easily formed by using standard passive optical splitters with symmetric or asymmetric splitting ratios, which would enable placing the secondary OLT unit into any possible position in a ring thus making the whole infrastructure less vulnerable. It is evident that a ring topology in case of PON would probably suffer several disadvantages, so it would not be very useful for standard PON applications, such as providing network connection for ordinary households and typical end-users, but its application for well protected specific situations in local area networks could enhance the overall security of the whole infrastructure.

Another problem of a PON with ring topology is a high value of insertion loss of passive optical splitters. That is why forming an optical distribution network with ring topology using only standard symmetric passive splitters would result into a very uneconomic solution, because only a 
limited number of network units could be connected in such case. However, using asymmetric passive optical splitters with splitting ratios calculated and optimized for specific scenario or tunable splitters, could significantly balance the attenuation in the whole infrastructure, thus enabling more ONUs to be connected [4].

This paper contains an initial idea of forming PON with ring topology consisting of two independent OLT units and passive optical splitters. The next part is focused on calculations of attenuation and its balancing for two scenarios of ring topology - using standard symmetric splitters, and using asymmetric splitters (or tunable splitters) with optimized but rounded splitting ratios, which can be easily manufactured and are widely available.

\section{PON WITH RING TOPOLOGY}

The ring topologies are usually used for backbone telecommunication networks (SDH, OTH, SONET), because they offer simple possibilities for efficient network protection (e.g. optical units, optical fibers). The situation in typical PONs is slightly different. Since the whole traffic and the whole network is controlled and operated from central OLT unit, its failure would certainly result in global PON malfunction [6]. As described in the text above and illustrated in Fig. 1, a star topology or a tree topology usually offers only one possible place for OLT, which makes it further vulnerable while both (primary and secondary) OLTs can be stroked at once. A possible solution is to use a ring type topology of optical distribution network. PON networks with a ring topology were already presented for the purpose of WDM-TDM long reach PON [5], but these applications are based on special optical network units (ONU) with optical switches and others nonstandard enhancements. However, the simple ring topology can be easily created by using only standard passive optical splitters. This solution is presented in Fig. 2.

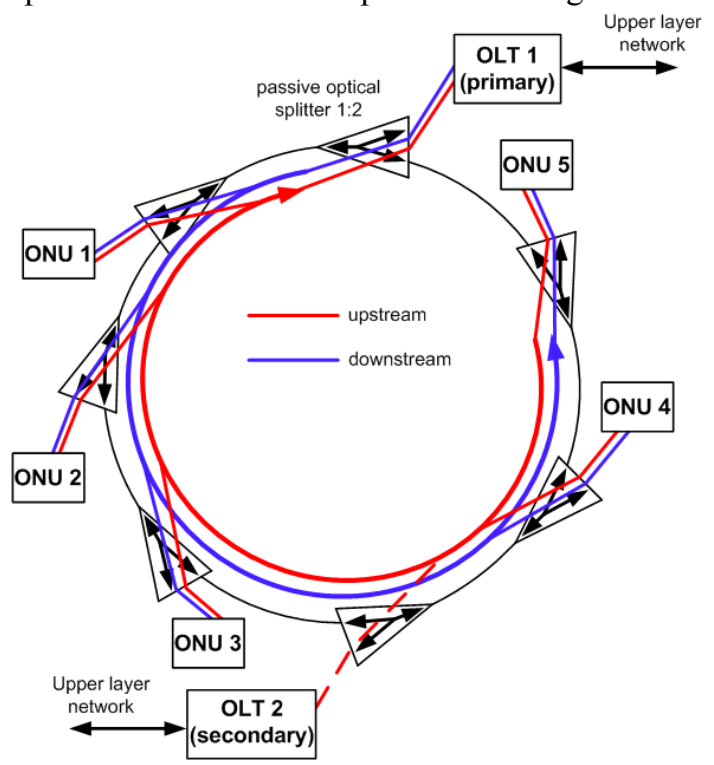

Fig. 2. Proposed ring-type PON network, initial state.

The proposed infrastructure contains two independent OLT units, which can be simply placed in any possible location within ring topology, however the symmetric situation with placing both OLT units exactly to the opposite positions of a ring (two identical halves) is optimal. This is the main advantage of proposed solution compared to the standard tree or star topologies - the relative independence of both (or even more) OLTs locations. While in case of a tree or star topology both OLT units (primary and secondary) can be stricken with a single attack or single global failure in one location, the OLTs in case of a ring topology are mutually almost independent and their elimination could be more difficult, because they can be located anywhere within the ring infrastructure. All optical units (OLTs, ONUs) are connected via standard passive optical splitters with splitting ratios 1:2. Assuming PLC (planar) type of splitters, their directivity and return loss is high enough to prevent crosstalk and other negative disturbing between neighboring units and transmission directions. Both OLT units are connected into the upper layer networks (backbone telecommunication networks) via standard Ethernet (metallic, optical), the more detailed description is discussed in the last section of this article.

In initial state, the primary OLT (OLT 1) acts as a main OLT and is providing all standard functions in PON network. The secondary OLT (OLT 2) is in warm-state backup and is only monitoring the upstream traffic for detecting the potential failures. If the malfunction or failure of the primary OLT appeared, the secondary OLT could switch to the main role and it could take over the whole traffic. This situation is illustrated in Fig. 3.

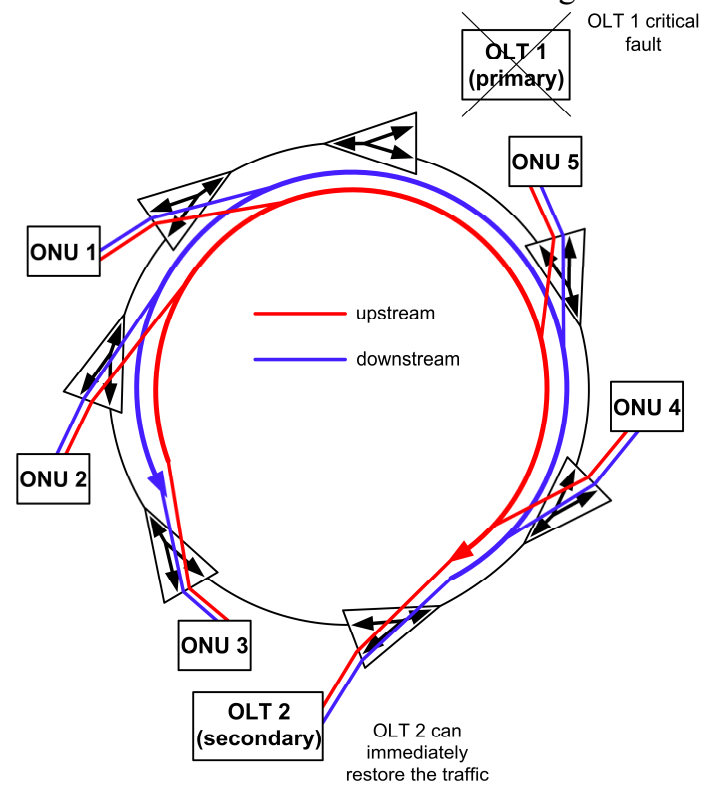

Fig. 3. If the critical fault of primary OLT occurs, the secondary OLT can restore the traffic.

By comparing Fig. 2 and 3 it is evident that both traffic directions can be easily adapted when the secondary OLT switches into the main role. It is also obvious that presented ring topology is basically a bus type topology with unused interconnection between the last ONU and the first section of a ring (OLT unit). Therefore it is necessary to perform detailed calculations and planning of attenuation and optical signal levels in all network nodes to prevent loops occurring of forthcoming optical signals. The calculations of splitting ratios and resulting attenuations for all passive splitters as well as practical example are presented in the next part of this article.

It would be therefore possible to use this proposed ring topology for designing more complex network 
infrastructures, e.g. two semi-dependent PON rings with two OLTs, as presented in Fig. 4.

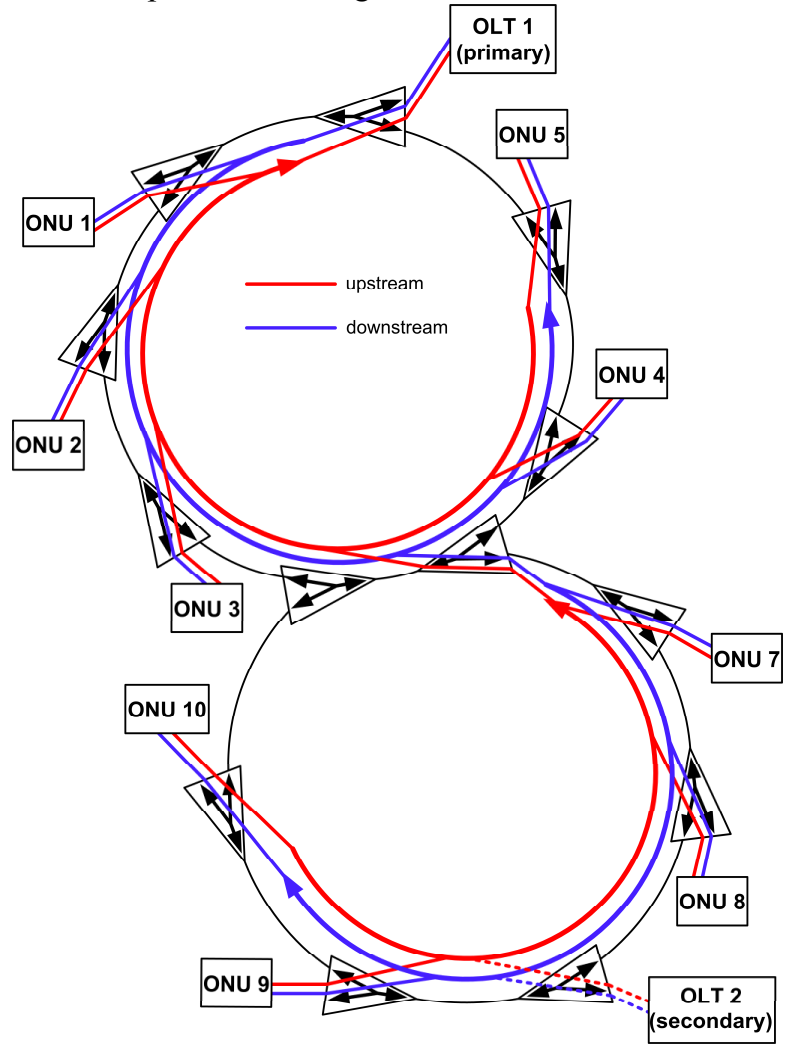

Fig. 4. Using ring topology for designing two semi-dependent protected PON networks.

In this scenario, each OLT can provide connection separately only to ONUs in each of two rings, or it would be also possible that one OLT acts as a primary unit for both rings and in case of its failure, the secondary OLT can switch to the primary role and take the data traffic over the whole infrastructure. For this reason, the splitting ratios of all passive splitters should be calculated and optimized to prevent loops occurring. If the optical level of looping optical signal is equal or lower than a minimum receivable signal on ONU side after passing throughout the whole ring, it would not influence the forthcoming traffic in downstream or upstream direction. Otherwise it would act as a disturbing signal increasing the noise level. Another possible solution would be the usage of band-stop and band-pass type filters, which would be placed at the ends of loops in both rings.

The splitting ratio of all tunable splitters should be optimized in all scenarios for enabling maximum ONUs to be connected into such infrastructure. This optimization could be performed using the algorithms presented in [4] and according to the lengths and number of optical fibers and ONUs. Practical application and example of results for a simple ring topology is presented within the next section of this article.

\section{OPTIMIZATION OF PON WITH RING TOPOLOGY}

Presented PON with ring topology in Fig. 2 and 3 is based on passive optical splitters with ratio $1: 2$ and two basic scenarios are possible - symmetric splitters (with uniform splitting ratio 50:50\%), and asymmetric tunable splitters with calculated and optimized but rounded splitting ratios.

\section{A. Symmetric and Asymmetric Passive Splitters}

The splitting ratio is a key parameter of the optical splitter. The splitting process itself is implemented by passive method, using elementary Y-junctions (a single Yjunction has a splitting ratio of $1: 2$ ), which can be made using short fibers fused together or using a planar technology. Subsequently, it is possible to obtain higher desired values of splitting ratio by cascading several elementary Y-junctions. Another important parameter of the passive optical splitter is the value of its total insertion loss $A_{C}$, which consists of the loss $A_{D}$ due to the process of dividing the input optical power into $N$ outputs and it also consists of the second part represented by a residual loss $A_{Z}$ [3]. The loss $A_{D}$ depends on the splitting ratio 1:N (it depends on the number of outputs $N$ ), the residual loss $A_{Z}$ represents the additional loss of fibers, connectors, fused joints and its irregularity, manufacturing tolerations, etc. The loss $A_{D}$ due to the dividing of input optical power for the $i$-th branch can be expressed as:

$A_{D i}=10 \log \left(\frac{P_{\text {in }}}{P_{\text {out }}}\right) \quad[d B ; W, W]$.

Where $A_{D i}$ is a loss given by splitting the optical signal with an input optical power $P_{i n}$, while the power of the optical signal on that output is $P_{\text {outi }}$. The summary output optical power is therefore given by summarizing all outputs:

$P_{\text {in }}=P_{\text {out }}=\sum_{i} P_{\text {out }_{i}} \quad[W ; W]$.

In case of a symmetrical splitter with only one Y-junction (1:2 splitter) and a uniform splitting ratio of the input optical power to its outputs $P_{\text {out }}=P_{\text {out } 2}=0.5^{*} P_{\text {in }}$, the splitting loss is $A_{D 1}=A_{D 2}=3.01 \mathrm{~dB}$. The asymmetric passive optical splitter with a non-uniform variable splitting ratio on its particular outputs can be made either with the fixed splitting ratio, or as a tunable optical splitter with the controllable splitting ratio [7]. The asymmetric splitter ( $\mathrm{Y}$-junction in this case) provides asymmetric ratio of optical powers on its outputs, therefore it is possible to express following conditions:

$P_{\text {out }_{1}} \neq P_{\text {out }_{2}} \quad[W ; W] ; A_{D_{1}} \neq A_{D_{2}} \quad[d B ; d B]$.

The initial condition of summary output power is still valid:

$P_{\text {in }}=P_{\text {out }}+P_{\text {out }} \quad[W ; W, W]$.

If a general attenuation $A_{l}$ is connected to the first branch of Y-junction and attenuation $A_{2}\left(A_{1} \neq A_{2}\right)$ to the second branch, it is possible to derive general formula for calculating desired splitting ratio in way, in which the resulting summary attenuations of both branches remain the same as:

$\frac{P_{\text {in }}}{P_{\text {out }}}=1+10^{\frac{A_{2}-A_{1}}{10}} \quad[W, W ; d B, d B]$

$\frac{P_{\text {in }}}{P_{\text {out }_{2}}}=\frac{1+10^{\frac{A_{2}-A_{1}}{10}}}{10^{\frac{A_{2}-A_{1}}{10}}} \quad[W, W ; d B, d B]$

Thanks to this equation, balanced (equal) attenuation of both branches of asymmetric splitter could be reached:

$A_{D_{1}}+A_{1}=A_{D_{2}}+A_{2} \quad[d B, d B ; d B, d B]$.

Finally, to obtain the resulting condition of optimizing 
(balancing) attenuations for specific application of asymmetric splitter, desired attenuations or levels of optical signals on its outputs are necessary. Detailed descriptions and evaluations of the formulas were already presented in [4], [8].

Basically, there are two different approaches to the practical applications of asymmetric passive optical splitters. One possibility is to use each splitter individually, with the splitting ratio being fully optimized for specific conditions, which further means to perform necessary calculations of required value of splitting ratio in the entire infrastructure first, and then to manufacture each splitter separately. The second option is the usage of tunable optical splitters.

\section{B. Calculations and Optimizations of Splitting Ratios for PON with Ring Topology}

The initial calculation is performed for standard symmetric passive optical splitters with uniform splitting ratios. Then, the following calculation is carried out for the asymmetric tunable passive splitters with specific splitting ratios, which are rounded in their percentage expressions. Common set of basic network parameters and parameters of typical optical elements are, as follows:

- Passive optical network is a GPON type with attenuation class $\mathrm{C}$, the interval of granted attenuation is therefore $A_{\text {min }}=15 \mathrm{~dB}, A_{\max }=30 \mathrm{~dB}$.

- The optical fiber used in the entire optical distribution network is specified according to the ITU-T G.652 recommendation as a $\mathrm{D}$ type fiber. Its attenuation coefficient at $1310 \mathrm{~nm}$ is therefore $\alpha=0.4 \mathrm{~dB} / \mathrm{km}$.

- The ring-type PON is proposed for special applications, such as a local protected network [4], therefore the distance between two passive optical splitters is $l_{l}=200$ $\mathrm{m}$, while the distance between the splitter and the optical network unit ONU (the length of a branch) is $l_{2}$ $=50 \mathrm{~m}$. These distances were considered to be the optimal lengths for following example of a local highspeed network.

- The connection of optical network termination OLT and optical network units ONUs to an optical network is realized with a connector with the insertion loss $A_{k}=0.2$ $\mathrm{dB}$. The residual loss of modern planar passive optical splitters should not exceed $0.5 \mathrm{~dB}$, therefore together with small compensation, it is possible to assume $A_{z}=$ $0.55 \mathrm{~dB}$. The attenuation reserve for the compensation of aging effect and temperature changes is $A_{r}=0.5 \mathrm{~dB}$.

Proposed ring-type PON is illustrated in the following schematic in Fig. 5.

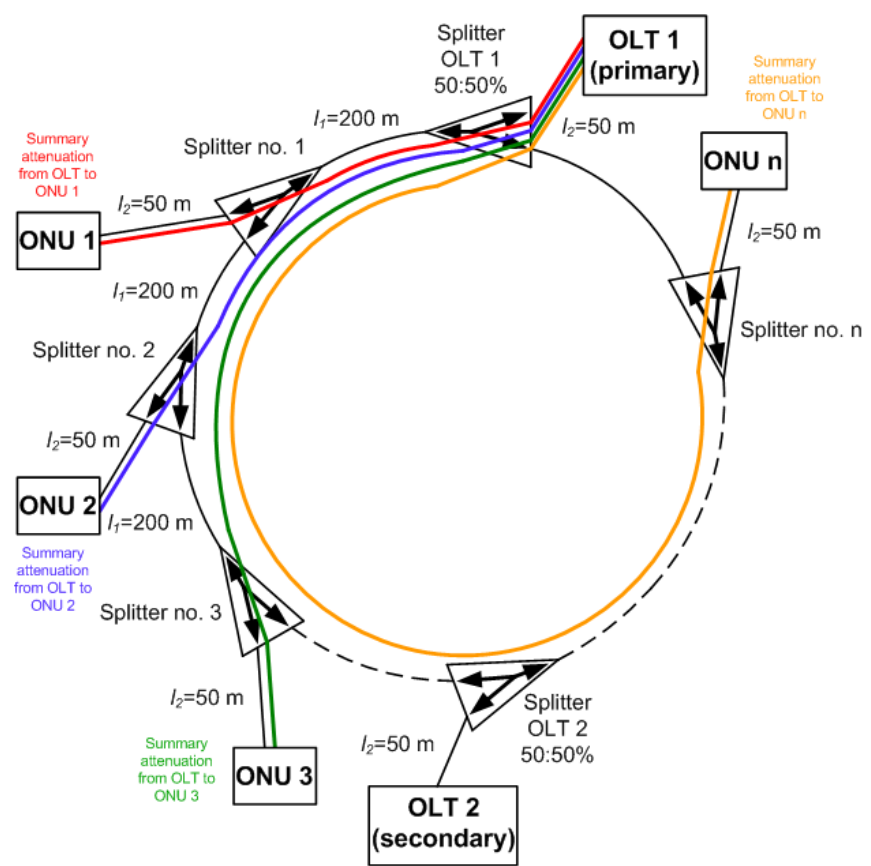

Fig. 5. Schematic illustration of proposed PON example with ring topology.

It is evident that one of the necessary functions of splitters connecting both OLTs is to prevent optical signal looping in a ring, thus isolating the last and first section of a ring. Therefore it is necessary to calculate the attenuation of the whole ring and to calculate the level of looping optical signal, which is transmitted through the last optical splitter. Because the whole infrastructure is symmetric, which means both sides of the ring contain the equal numbers of optical splitters (ONU units), the optimum splitting ratios of the splitter connecting primary OLT (OLT 1) should be 50:50\% and the same splitter should also connect the secondary OLT 2 unit. Symmetrical splitters (50:50\%) in case of connecting OLT units are optimal, because in case of OLT switching, the direction of optical signal in a ring is adapted towards a new situation and for maximum flexibility of the whole infrastructure, the symmetrical splitters for connecting OLT units should be used.

1) Calculations in Case of Symmetric Splitters

First, the calculation of attenuation was performed for PON with ring topology with parameters described above, using symmetric passive optical splitters with uniform splitting ratio 50:50\%. Graph in the following Fig. 6 presents the results of calculation of attenuations from OLT to all ONUs.

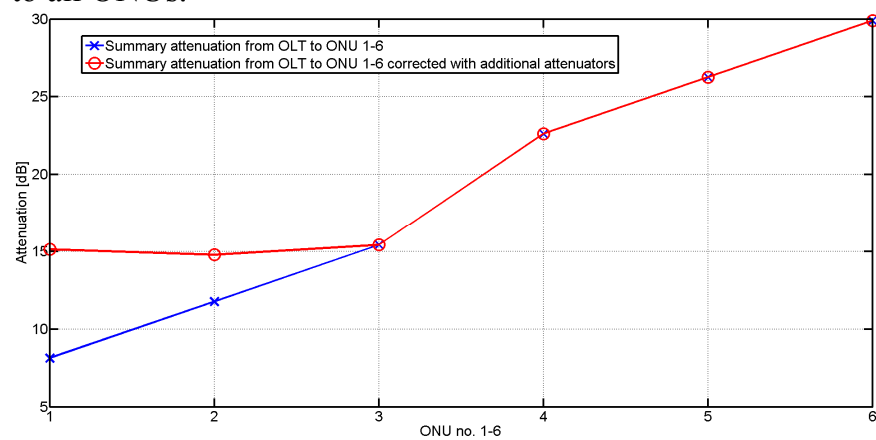

Fig. 6. Calculated attenuations from OLT to each ONU and the correction with using additional attenuators.

Based on the results, it can be concluded that with regard to maximum attenuation $A_{\max }$ for the $\mathrm{C}$ class of GPON it is possible to use only 6 standard passive splitters with 
symmetric splitting ratio, which means only 6 optical units (ONUs) can be connected to the optical infrastructure, as illustrated in Fig. 6. Moreover, some network ring branches do not meet the minimum attenuation $A_{\min }$ specified for $\mathrm{C}$ class GPON. Therefore it is necessary to provide the correction by using additional attenuators for increasing their attenuation, which is also presented in Fig. 6. It is also necessary to calculate and check the overall attenuation of the whole ring infrastructure, so the optical signal passing throughout the ring and appearing on its beginning (interfering signal) is attenuated enough to not interfere with main transmission. This value of SIR (Signal to Interference Ratio) can be expressed as:

$$
S I R=\frac{2\left(1-N_{M}\right)}{H_{0} N_{M} H_{\min }}[d B] .
$$

Where $M$ is the number of ONU units in a ring network, $N_{M}$ represents the transmission function of the passive splitter of $M$-th ONU, $H_{0}$ stands for the basic transmission function of a network segment between two passive splitters and $H_{\min }$ is a minimum transmission function satisfying the condition of minimum receivable optical signal the last ONU unit in the ring topology. The value of $N_{M}$ in case of all symmetrical splitters is $N_{M}=0.5 . H_{0}$ can be expressed as:

$H_{0}=10^{-\frac{A_{0}}{10}} \quad[-; d B]$.

Where $A_{0}$ can be calculated using attenuation coefficient $\alpha$ and the summary length $l$ of each segment of proposed ring topology. The general formula for calculating minimum transmission function $H_{\min }$ of ring network for $M$-th $\mathrm{ONU}$ unit satisfying the $A_{\min }$ value can be expressed as:

$$
H_{\min }=\frac{1}{2} H_{0}^{M+1+j} \prod_{i=j}^{M} N_{i} \cdot\left(1-N_{j}\right) \prod_{k=0}^{j-1} N_{k}^{2} .
$$

Where $N_{i}, N_{k}$ stands for the cascaded transmission functions of $M$ passive optical splitters in the ring topology towards the ONU units and fiber segments.

The value of the SIR parameter in the first scenario with symmetric passive splitters is approx. $33.32 \mathrm{~dB}$, which is fully suitable for proper function of proposed PON network.

\section{2) Optimization of Ring Topology Using Asymmetric}

\section{Tunable Splitters}

The next calculation considers the usage of asymmetric tunable passive splitters with a non-uniform splitting ratio, which can be calculated and optimized. Nowadays, tunable optical splitters are still not very common, while they usually require external power source and management and are more expensive than standard passive optical splitters. However, several technologies have been already proposed to produce tunable optical splitters with various types of their internal structure and parameters. Different methods of achieving non-uniform tunable splitting ratio have been utilized in [7], [9]. The splitting ratios of these tunable splitters in proposed ring topology were individually calculated for each passive optical splitter according to the equations (4), (5) and they were rounded in their percentage expression. The initial idea of a successful optimization process is achieving balanced attenuation values for all ring paths between OLT and each ONU. These values should approach, in an ideal state, the
$A_{\max }$ value of $30 \mathrm{~dB}$ for selected variant GPON class C. Following graph in Fig. 7 presents the results of calculated values of splitting ratios for all passive splitters.

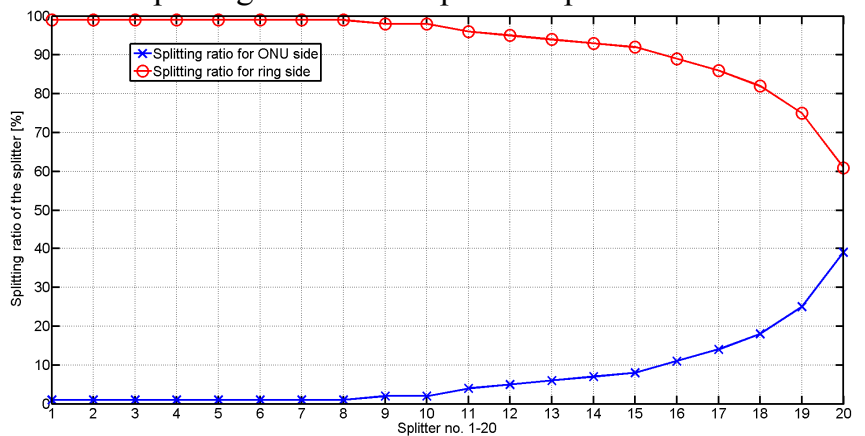

Fig. 7. Calculated splitting ratios of individually optimized splitters.

Through optimization, the balanced values of attenuation are reached in all sections of designed ring topology. Therefore, it is possible to connect 20 passive splitters $(20$ ONUs) to this optical infrastructure, which is significantly more than in case of symmetric splitters. The values of summary attenuation for each ring branch (paths from OLT to each ONU) as well as the attenuation of all sections of proposed ring topology are presented in next Fig. 8.

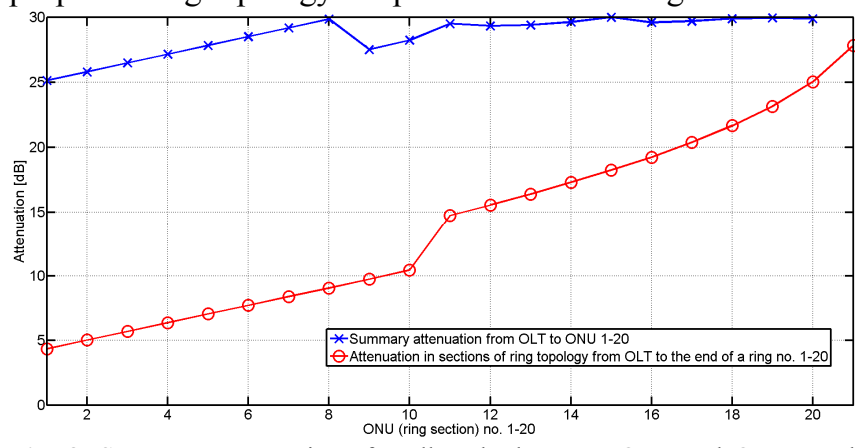

Fig. 8. Summary attenuations for all paths between OLT and ONUs and attenuations for all sections of ring topology.

In case of accurate optimization, all branches (paths) between OLT and ONUs should reach the $A_{\max }$ value of selected PON type and attenuation class. Due to the rounding of calculated splitting ratios in their percentage expressions, several branches are not perfectly and accurately optimized, although the attenuations of these branches are close enough to the $A_{\max }$ value and they meet the requirements for the minimum value of $A_{\min }$ and thus it is not necessary to use additional attenuators. It is obvious that in case of switching both OLT units (switching primary and secondary unit), it will be also necessary to retune splitting ratios of all used splitters, that is why using tunable splitters is proposed. It is also necessary to calculate the attenuation of interfering optical signal in the same way, as it was calculated in the previous simulation of symmetric splitters using formula (7). The value of SIR in this case is:

$S I R=31.37 d B$

This value is, again, fully suitable to guarantee error-free transmissions in a ring.

\section{PRACTICAL APPliCABILITY OF Results}

It is evident that a ring topology in case of PON would probably suffer several disadvantages, so it would not be very useful for standard PON applications, such as providing network connection for ordinary households and typical endusers, but its application for well protected specific 
situations in local area networks could enhance the overall security of the whole infrastructure. Previously presented calculations of physical parameters (attenuation) in case of PON with ring topology illustrated that proposed infrastructure could be possibly used for a real application. It is evident that the proposed ring topology is applicable and could be realized even with the use of standard symmetric splitters $(50: 50 \%)$ for some specific situations. In the first presented scenario, only a limited number of 6 ONU units could be possibly connected into this ring topology to meet the maximum attenuation given for the specific PON type (GPON class $\mathrm{C}$ in this case). However, even such solution could be practically realized and applied for some specific scenario of well protected local PON network, when higher network reliability and protection against OLT unit failure is required.

To increase the number of connected ONU units, tunable asymmetric splitters are necessary to use, as it is presented in the previous section with calculation of optimized splitting ratios of tunable splitters. The previous example illustrated that this scenario could result into connecting of $20 \mathrm{ONU}$ units, which is significantly higher than in previous case with symmetric splitters. The tunable splitters are not usually common components today, however, several theoretical proposals have been already presented [7], [9], [10], so these splitters will be probably available soon. It would be also possible to use asymmetric splitters with fixed splitting ratio (non tunable), however, in case of OLT units switching, the attenuation of optical signal in case of some ONU units would not meet the demands given in a specific recommendation.

Future research in this area should be focused on practical realization and testing of proposed ring topologies to verify its parameters and functions. Another function that needs to be verified is the switching mechanism of OLT units. These units are usually connected via Ethernet (metallic, optical) to the backbone telecommunication networks, therefore there are two possible scenarios:

- Switching is an internal function of OLT units,

- Switching would be performed by a network node (element) of a higher layer network.

While the first option requires a proprietary internal protocol (or rules) implemented in the OLT units, the second scenario could be used on standard OLT units without any enhancements. The time necessary for switching the OLT units could be shorter in the first scenario, because it could be triggered by OLT units themselves in case of low level of optical signal detection (OLT 2 units passively monitors the traffic and in case of low or no signal detection, it could switch to the main role). The second scenario would be possibly based on using a network element in upper layer network, which again in case of no incoming traffic from OLT 1 unit would activate the backup OLT 2 unit. Again, a proprietary protocol would be necessary or it would be also possible to use standard spanning-tree protocol, which is often applied in Ethernet networks to prevent loops occurring and which can be also configured for dynamic reconfiguration of network. That is why in case of some OLT unit failure the upper node could quickly switch the traffic via the backup one. All these situations are needed to be verified and properly tested. however, the necessary equipment was not available at the present time. Therefore these tasks are to be focused on and to be evaluated through further research.

\section{CONCLUSION}

This article proposes an innovative method for protection of PON networks, especially its central optical unit - OLT. This method is focused on forming of PON with ring topology using passive optical splitters and neither special enhancements nor optical switches are necessary. The main idea is based on the possibility of placing both OLT units (primary and secondary) on the opposite sides of the ring. This could increase the resistance of whole PON infrastructure against failures of a single central OLT unit, because secondary OLT operates in a warm-state backup and could restore the traffic. By using this method, more complex double-rings or multiple-rings topologies can be created and they can offer several specific benefits for network protection or management.Standard symmetric passive splitters with uniform splitting ratio offer only limited possibilities for PON with ring topology, therefore due to the optimization process and using asymmetric passive splitters, the number of connected ONUs in a ring topology could be significantly increased. However, in case of switching primary and secondary OLT units, the splitting ratios of all used splitters have to be optimized and tuned again to meet the requirements for a new situation.

\section{REFERENCES}

[1] ITU-T, "G.987.1 - 10-Gigabit-capable passive optical network (XGPON) systems: Definitions, Abbreviations, and Acronyms," ITU-T, January 2010. Available http://www.itu.int/rec/T-REC-G.987201001-I.

[2] IEEE, "IEEE Standard 802.3av-2009, Amendment 1: Physical Layer Specifications and Management Parameters for $10 \mathrm{~Gb} / \mathrm{s}$ Passive Optical Networks," IEEE 802.3av 10G-EPON Task Force. [online], September 2009. Available http://www.ieee802.org/3/av/.

[3] C. F. Lam, Passive Optical Networks: Principles and Practice. Academic Press of Elsevier Inc., Burlington, USA. 2007.

[4] P. Lafata, J. Vodrážka, "Application of Passive Optical Network with Optimized Bus Topology for Local Backbone Data Network," Microwave and Optical Technology Letters. 2011, vol. 53, no. 10, p. 2351-2355.

[5] S. Dong-Min, J. Eui-Suk, K. Byoung-Whi, "A simple passive protection structure in a ring-type hybrid WDM/TDM-PON," 11th International Conference on Advanced Communication Technology. ICACT 2009, February 2009, p. 447-449.

[6] C. M. Machuca, J. Chen, L. Wosinka, "PON protection architectures achieving total cost reduction," Conference and Exhibition (ACP) of Communications and Photonics, 2010 Asia, December 2010, p. $707-$ 708 .

[7] S. Xia, Q. J. Zeng, J. Wang, H. Zhao, H. Chi, Y. Wang, F. Liu, F., X. Zhu, "Tunable optical splitter technology," SPIE Proceedings, Vol. 4870-70, SPIE, July, 2002.

[8] P. Lafata, J. Vodrážka, "Návrh pasivních optických sítí s optimálními rozbočovacími poměry," Elektrorevue. 2010, vol. 13, no. 67, p. 67-167-8. Available: http://elektrorevue.cz/cz/clanky/komunikacnitechnologie/0/navrh-pasivnich-optickych-siti-s-optimalnimirozbocovacimi-pomery/.

[9] Z. Yun, L. Wen, Ch. Long, X. Qingming, "A 1x2 Variable Optical Power Splitter Development," Journal of Lightwave Technology, vol. 24, no. 3, March 2006.

[10] X. Tang, J. Liao, H. Li, L. Zhang, R. Lu, Y. Liu, "Design and analysis of a novel tunable optical power splitter," Chinese Optics Letters, vol. 9 , no. 1, January 2011 